\title{
SIMULACIÓN NUMERICA DEL PROCESO DE INFECCION TRATAMIENTO DEL VIH1 Y SU CONTROL BAJO LA ACCIÓN DE ANTIRRETROVIRALES
}

\author{
NUMERICAL SIMULATION PROCESS AND HIV INFECTION \\ CONTROL IN THE ACTION ANTIRETROVIRALS
}

\author{
Irla Mantilla ${ }^{1}$, Carlos Masgo ${ }^{2}$
}

\begin{abstract}
RESUMEN
En este trabajo se considera la propuesta, desarrollo y simulación numérica de un sistema que modela el comportamiento del tratamiento del VIH-1, utilizando antirretrovirales. El sistema definido para tres variables dependientes de la variable $t$, denotadas por $X(t)=\left(x_{1}(t), x_{2}(t)\right.$, $\left.x_{3}(t)\right)$ representan a la cantidad de Linfocitos $T$ "Helpers" (CD4), cantidad de Linfocitos $T$ Citotóxicos (CD8) y Carga Viral del proceso de infección del VIH-1 en cualquier instante de tiempo $t$, respectivamente. El sistema conformado por un conjunto de Ecuaciones Diferenciales Ordinarias No lineales, cuyo dominio existencial del sistema representa el tiempo de evaluación del proceso de infección y eliminación del virus en un paciente con VIH-1. Este conjunto de estados del Sistema Dinámico No lineal, asociado a las condiciones de valor inicial es denominado Problema de Cauchy. Existen pocos estudios relacionados a la solución de este sistema, de los encontrados algunos se reducen al estudio en dos variables y otros sin la obtención de la solución explícita. En el presente trabajo se contribuye con el estudio del sistema para tres variables, y un análisis cualitativo y cuantitativo que comprende: linealización, diagrama de fase, estabilidad cualitativa, existencia y unicidad de solución analítica, donde se prueba una equivalencia de la solución del sistema no lineal a la del sistema linealizado. La equivalencia está basada en el Teorema de Grobman - Hartman y se encuentra la solución explícita mediante el método de Runge Kutta de 4to orden. De este modo se obtienen los resultados del modelo y cuya convergencia, está garantizada por la consistencia y estabilidad del esquema numérico.
\end{abstract}

Palabras clave.-Tratamiento antirretroviral, Modelamiento matemático VIH, Sistema dinámico no lineal, Método de Runge Kutta de cuarto orden.

\begin{abstract}
This paper considers the proposal, development and numerical simulation of a system that models the behavior of the treatment of HIV-1 using antiretrovirals. The system defined for three dependent variables of the variable $t$, denoted by $X(t)=\left(x_{1}(t), x_{2}(t), x_{3}(t)\right)$ represent the amount of T Lymphocytes "Helpers" (CD4), number of Cytotoxic T Lymphocytes (CD8) Viral load and infection process of HIV-1 at any time $t$, respectively. The assembly consists of a Nonlinear Ordinary Differential Equations system whose existential domain represents time system evaluation process of infection and viral clearance in a patient with HIV-1. This set of states of Nonlinear Dynamic System, associated with the initial value condition is called Cauchy problem. There are few studies related to the solution of this system, found some of the study are reduced to two variables and others without obtaining the explicit solution. Equivalence of the solution of the system linearization, phase diagram, qualitative stability, existence and uniqueness of analytic solution, where test: In this paper contributes to the study
\end{abstract}

La Revista Científica TECNIA protege los derechos de autor bajo la Licencia 4.0 de Creative Commons: Attribution 4.0 International (CC BY 4.0)

${ }^{1}$ Dra. del Laboratorio de Simulación e Investigación Numérica de la Facultad de Ciencias de la Universidad Nacional de Ingeniería, ${ }^{2}$ Dr. del Laboratorio de Simulación e Investigación Numérica de la Facultad de Ciencias de la Universidad Nacional de Ingeniería. 
of the system for three variables, and qualitative and quantitative analysis comprising nonlinear the linearized system. The equivalence is based on Theorem Grobman - Hartman and explicit solution by the Runge Kutta 4th order, thus the model results and whose convergence is obtained is, is guaranteed by the consistency and stability of the numerical scheme.

Key words.- Antiretroviral therapy, HIV mathematical modeling, Nonlinear dynamic system, Runge Kutta method of fourth order.

\section{INTRODUCCIÓN}

Los Sistemas Dinámicos son modelos Matemáticos que se aplican a distintas ramas de la Ciencia, Ingeniería, Biología, Medicina etc. En el presente trabajo analizamos un tipo de Sistemas Dinámicos no Lineales Rígidos aplicado a un modelo Matemático del VIH-1 [1], garantizaremos la existencia y unicidad de solución, analizaremos el Sistema no lineal aplicando el Teorema de Grobman- Hartman que nos garantiza la existencia de un homeomorfismo entre campos definidos sobre conjuntos con singularidades hiperbólicas con la finalidad de preservar las orientaciones de las órbitas en su respectivo plano de fase, la existencia de una equivalencia topológica y el desarrollo de estos tipos de sistemas usando un método numérico de Runge Kutta de cuarto orden en tres dimensiones.

El contenido de este trabajo está conformado por el siguiente desarrollo: modelaremos el estado del Sistema no Lineal, plantearemos el problema según la geometría del dominio del estudio, espacio de definición del problema y su condición inicial del Problema de Valor Inicial (P.V.I).

Estudiaremos el análisis cualitativo de un Sistema Dinámico no Lineal mediante: el Teorema de Grobman - Hartman, una aplicación de dicho Teorema al modelo matemático del VIH-1 para el caso $\mathrm{u}=0$ ( $\sin$ tratamiento [2]), $\mathrm{y} \quad u \neq 0$ (con tratamiento [3]).

Analizaremos cuantitativamente la solución del Sistema no lineal empleando un método numérico para resolver el problema de Sistemas Dinámicos Rígidos No Lineales, este método es el método de Runge Kutta de cuarto orden para tres variables dependientes del tiempo, al igual que se implementa su algoritmo de simulación y se analiza, su convergencia.
Los resultados numéricos incluyen gráficos de las soluciones en cada componente y en ambos casos de estudio, analizaremos el comportamiento del CD4 [3] y CD8 [4] al año de iniciar el tratamiento a los pacientes y se predice el tiempo en el que un paciente alcanzaría la cantidad de células de una persona sana, es decir, cuando la carga viral es nula [5].

\section{MODELO MATEMÁTICO}

Para nuestro objetivo representamos por $f$ el Campo que define el comportamiento de variación sobre un dominio espacial $\Omega$ de las variables determinadas por el sistema de coordenadas cartesianas $\mathrm{X}(\mathrm{t})$, donde: $\mathrm{X}(\mathrm{t})=\left(\mathrm{x}_{1}(\mathrm{t}), \mathrm{x}_{2}(\mathrm{t}), \mathrm{x}_{3}(\mathrm{t})\right)$; $\mathrm{x}_{1}, \mathrm{x}_{2} \mathrm{y} \mathrm{x}_{3}$ son funciones no lineales dependientes del tiempo, y representan a la evaluación de la cantidad de linfocitos al que designamos por las siglas CD4, la evaluación de la cantidad de linfocitos citotóxicos por las siglas CD8 y la cantidad de Carga Viral respectivamente, según [6], se puede modelar, considerando como dominio de estudio, un conjunto abierto

$\Omega \subseteq[0, N] \times[0, N]^{3}, \subseteq I R \times \mid R^{3}$ tal que el estado del flujo $X(t)$, representado por una, Trayectoria en $I^{3}$, [7], denominada órbita es la solución del Sistema Dinámico No lineal que a continuación se detalla:

Sean $f=\left(f_{1}, f_{2}, f_{3}\right)$ una aplicación de clase $\left[\mathrm{C}_{1}\right.$

$(\Omega)]^{3}$ denominado Campo [8], X una función de clase $\left[\mathrm{C}^{1}(\Omega)\right]^{3}, \mathrm{t} \in[0, T, \mathrm{~T}>0$,

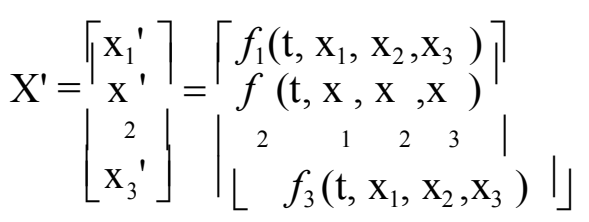

En particular 


$$
\begin{aligned}
& X^{\prime}=\left(\begin{array}{ll}
\mathrm{x}_{1} & \\
\mathrm{x}^{\prime} & 1 \\
\mathrm{x}_{2} & \left.\right|_{3} ^{\prime}
\end{array}\right)=
\end{aligned}
$$

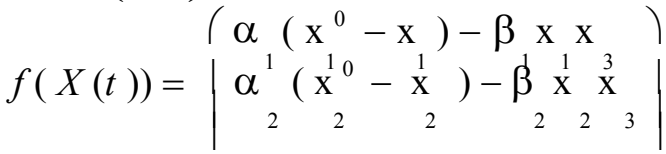

$$
\begin{aligned}
& \left(\beta_{3} \mathrm{x}_{1} \mathrm{x}_{3}-\beta_{4} \mathrm{x}_{2} \mathrm{x}_{3}-u\right)
\end{aligned}
$$

Donde $\mathrm{x}_{1}(\mathrm{t})$ es la Concentración de linfocitos $\mathrm{T}$ "Helpers" (CD4) (células $/ \mathrm{mm}^{3}$ ) en cualquier instante $\mathrm{t}, \mathrm{x}_{2}(\mathrm{t})$ es la Concentración de linfocitos $\mathrm{T}$ Citotóxicos (CD8) (células $/ \mathrm{mm}^{3}$ ) en cualquier instante $\mathrm{t}$.

Estas magnitudes caracterizan las variables de estudio del modelo matemático denominado Proceso de Infección - Tratamiento, del VIH-1.

\section{Notación:}

$\mathrm{x}_{1}^{\prime}=\frac{d \mathrm{x}_{1}}{d t}, \mathrm{x}_{2}^{\prime}=\frac{d \mathrm{x}_{2}, \mathrm{x}^{\prime}=}{d t}=\frac{d \mathrm{x}_{3}}{d t}$

$\alpha_{1}$ : decrecimento natural de los linfocitos T CD4

$\alpha_{2}$ : decrecimiento natural de los linfocitos T CD8

$\beta_{1}$ : Efectividad de infección viral

$\beta_{2}$ : Producción de linfocitos TCD8 en el proceso de infección

$\beta_{3}$ : Producción de viriones basado en linfocitos TCD4

$\beta_{4}$ : Decrecimiento de viriones debido a linfocitos TCD8

$u$ : Parámetro de control del tratamiento con antirretrovirales

\section{Condición inicial:}

$$
\left(\mathrm{x}_{1}^{0}, \mathrm{x}_{2}^{0}, \mathrm{x}_{3}^{0}\right)=(1000,550,0)
$$

El dominio de estudio es el tiempo representado por:

$\mathrm{t} \in\left[0, T, \mathrm{~T}>0\right.$, las variables de estado $\mathrm{x}_{1}(\mathrm{t})$, $\mathrm{x}_{2}(\mathrm{t}) \mathrm{y} \mathrm{x}_{3}(\mathrm{t})$ son las variables de estado del Sistema Dinámico no Lineal.

$\mathrm{x}_{3}(\mathrm{t})$ : Carga viral en cualquier instante $\mathrm{t}$

Una prueba de carga viral en plasma (que también se conoce por las siglas PCV) mide la cantidad de Virus de Inmunodeficiencia Humana (VIH) está presente en la sangre. A esta cantidad presente en la sangre se denomina "carga viral" [7].

Cuanto menor sea la carga viral, menor será la cantidad del VIH.

Los resultados de las pruebas de PVC suelen indicarse como "copias por mililitro (ml)" de sangre. Las pruebas de PVC miden cuánto Acido Ribonucleico (ARN) del VIH hay en una muestra de sangre.

El ARN es el "molde" que el VIH usa para fabricar más cantidad del virus. Cada VIH lleva 2 copias de ARN. Esto significa que si el informe de la prueba indica que hay 20,000 copias de ARN del VIH por ml, el paciente tiene 10,000 partículas de virus por cada $\mathrm{ml}$ de sangre. Esta unidad es denotada por $\mathrm{N}^{\circ}$ de veces $\times 10^{7}$ copias $/ \mathrm{ml}$ ó $\mathrm{N}^{\circ}$ de veces $\times 10^{3}$ copias $/ \mathrm{mm}^{3}$. Por ejemplo las siguientes condiciones iniciales en una persona normal son:

$\mathrm{x}_{1}{ }^{0}=1000$ células $/ \mathrm{mm}^{3}$ la concentración inicial de CD4.

$\mathrm{x}_{2}{ }^{0}=550$ células $/ \mathrm{mm}^{3}$ la concentración inicial de CD8.

$\mathrm{x}_{3}{ }^{0}=0$ : Es decir ausencia de infección del VIH-1. Entonces el decrecimiento de linfocitos:

$\alpha_{1}=0.25 /$ año

$\beta_{1}=50 \mathrm{ml} / 10^{7}$ copias/año

$\alpha_{2}=0.251 /$ año $\beta_{2}=10 \mathrm{ml} / 10^{7}$ copias $/$ año

$\beta_{3}=0.01 \mathrm{~mm}^{3} /$ células/año).

$\beta_{4}=0,0045 \mathrm{~mm}^{3} /$ células/año).

La entrada de control en la terapia con agentes antirretrovirales preescrita al paciente, se interpreta como una tasa de eliminación viral provista por los fármacos $\times 10$ copias $/ \mathrm{ml}$. día.

\section{ESTUDIO CUALITATIVO DEL PROBLEMA}

En esta parte vamos a ver las propiedades cualitativas de sistema dinámico del VIH-1 planteado en la ecuación (1) aún sin conocer explícitamente la solución del sistema para ello aplicaremos el Teorema de Grobman-Hartman que 
garantiza que el sistema lineal y no lineal son cualitativamente y topológicamente equivalentes [8 y 9] alrededor de los puntos de equilibrio.

\section{Obtención de los puntos de equilibrio (PE)}

Los puntos de equilibrio se obtienen de la ecuación (1), haciendo

$$
f(X(t))=0 \text {, es decir, } \mathbf{P E}=\left(\mathrm{x}_{1}{ }^{*}, \mathrm{x}_{2}{ }^{*}, \mathrm{x}_{3}{ }^{*}\right)
$$

I) $\quad$ Con $u=0$ (sin tratamiento):

Encontramos 2 puntos de equilibrio a partir de la condición inicial de una persona sana, es decir CI $=(1000,550,0)$, entonces:

A) $\mathrm{Si}$ no hay infección.- se tiene que CD4 (concentración normal de linfocitos T, CD8 de una persona sana, y la carga viral, coincide con el punto de equilibrio PE, es decir:

$\mathbf{P E}=\left(\mathrm{x}_{1}{ }^{*}, \mathrm{x}_{2}{ }^{*}, \mathrm{x}_{3}{ }^{*}\right)=\mathrm{CI}=(1000,550,0)$

B) Proceso de infección.- Significa que se inicia cuando disminuye la cantidad de linfocitos TCD4 y aumenta la de CD8, es decir el punto de equilibrio está dado por:

$$
\begin{gathered}
x_{1}^{*}=\frac{\beta_{2} \beta_{1} x^{0}+\beta \beta_{1} \alpha_{2} x^{0}}{\beta_{3}\left(\beta_{1} \alpha_{2}+\beta_{2} \alpha_{1}\right)}=372.92 \\
x_{2}^{*}=\frac{\beta_{2} \beta_{3} \alpha_{4} x_{1} x_{1}+\beta_{2} \beta_{1} \alpha_{2} \alpha_{1} x_{2}}{x_{2}}=828.7 \\
x_{3}^{*}=\frac{\alpha_{2} \alpha_{3}\left(\beta_{3} x_{1}^{0}-\beta_{4} x_{2}^{0}\right)}{\beta_{4} \beta_{3} \alpha_{1} x_{1}^{0}+\beta_{1} \beta_{4} \alpha_{2} x_{2}^{0}}=8.8 \times 10^{4}
\end{gathered}
$$

Como se puede observar el punto de equilibrio de una persona con infección no coincide con la condición inicial de una persona sana, es decir:

$\mathbf{P E}=\left(\mathrm{x}_{1}{ }^{*}, \mathrm{x}_{2}{ }^{*}, \mathrm{x}_{3}{ }^{*}\right)=\left(372.92,828.7,8.8 \mathrm{x} 10^{4}\right)$.

Nuestro interés es analizar la estabilidad de la solución cualitativa en este estado de infección, para ello, hacemos lo siguiente:

Linealizando la ecuación (1), y al evaluar en los puntos de equilibrio (puntos singulares del sistema) para obtener los valores propios de la matriz A, asociada al sistema y se concluye lo siguiente:

$$
A=\left.\frac{\partial f}{\partial x_{i}}\right|_{x^{i}=x^{*}}=\left[\begin{array}{ccc}
-\alpha_{1}-\beta_{1} x_{3}^{*} & 0 & -\beta_{1} x^{*} \\
0 & -\alpha+\beta x^{*} & \beta x^{*} \\
\beta x^{*} & -2 \beta x^{* 2} & \beta x^{* 2}-\beta x^{*} \\
33 & 43 & 3 y_{2}
\end{array}\right]
$$

y se obtienen los siguientes valores propios reales:

- $0.25,-0.25$ y 7.525 , con los cuales se puede observar que dicho comportamiento cualitativo resulta ser un punto silla, es decir, es inestable. (Punto inicial y crítico 2) de una persona infectada) Infección en $\left(372,92 ; 828,7 ; 8,4 \times 10^{4}\right)$ se obtienen los siguientes autovalores $(-0,255$, $0,29-1,33 \mathrm{j},-0,29+1,33 \mathrm{j})$, el cual es un Nodo Foco Estable. La solución del sistema no lineal es topológicamente conjugada o equivalente topológicamente a la solución del sistema linealizado, cuya matriz asociada está dada por la matríz jacobiana $\mathrm{A}=\frac{\partial f}{\partial x}\left(x^{*}, x^{*}, x\right)^{*}$.

$$
\partial x \quad 1 \quad 2 \quad 3
$$

A continuación se detalla en las Tablas 1 y 2 los procesos de infección y No infección

Tabla 1. Punto de equilibrio y aproximaciones lineales cuando $(u=0)$.

\begin{tabular}{lll}
\hline Condición & $\begin{array}{c}\text { Punto de } \\
\text { equilibrio }\end{array}$ & $\begin{array}{c}\text { Aproximación } \\
\text { (autjugiflres) }\end{array}$ \\
\hline $\begin{array}{l}\text { No } \\
\text { infección }\end{array}$ & $(1000,550,0)$ & $-0,25,-0,25,7,525$ \\
& & \\
Infección & $(372,92,828,7$, & $-0,255$, \\
& $\left.8,4 \times 10^{4}\right)$ & $-0,29 \pm 1,33 \mathrm{j}$ \\
& & \\
\hline
\end{tabular}

Puntos de equilibrio con $(\mathrm{u} \neq \mathbf{0})$

Encontramos 2 puntos de equilibrio:

A) No infección.- Concentración normal de linfocitos $\mathrm{T}$

$\left(\mathrm{x}_{1}{ }^{*}, \mathrm{x}_{2}{ }^{*}, \mathrm{x}_{3}{ }^{*}\right)=(1000,550,0)$ 
B) No infección.-

$$
\begin{aligned}
& \mathrm{x}_{1}{ }^{*}=549, \\
& \mathrm{x}_{2}{ }^{*}=1007, \\
& \mathrm{x}_{3}{ }^{*}=0 .
\end{aligned}
$$

El Jacobiano está dado por

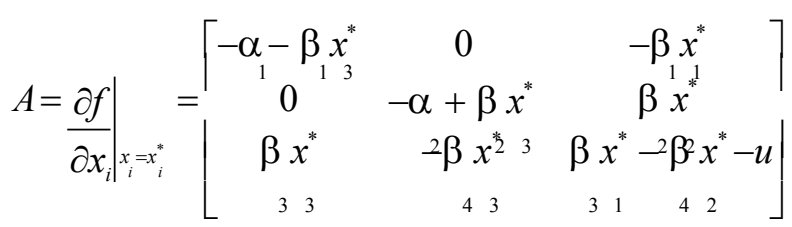

Al evaluar estos puntos singulares y obtener los valores propios de A se concluye:

No infección en $(1000,550,0)$ se obtienen los siguientes autovalores: $-0,25,-0,25$ y $-0,075$ es un punto atractor (Estable).

No infección en $(1007,549,0)$ se obtienen los siguientes autovalores: $-0,255,-0,25,-5 \times 10^{-4}$ es un punto atractor, es decir, Estable.

La solución del sistema no lineal es topológicamente conjugado al sistema lineal cuya matriz asociada es $\mathrm{A}=\frac{\partial f}{\partial x}\left(x_{1}^{*}, x_{2}^{*}, x_{3}^{*}\right)$.

Tabla 2. Punto de equilibrio y aproximaciones lineales cuando $(u \neq 0)$.

\begin{tabular}{llll}
\hline Condición & $\begin{array}{l}\text { Punto de } \\
\text { equilibrio }\end{array}$ & $\begin{array}{l}\text { Aproximación } \\
\text { lineal } \\
\text { (autovalores) }\end{array}$ \\
\hline No infección & $(1000,550,0)$ & $\begin{array}{l}-0,25,-0,25, \\
0,075\end{array}$ & - \\
& & 0.9 &
\end{tabular}

$\begin{array}{ll}\text { No infección } \quad(1007,549,0) \quad \begin{array}{l}-0,25,-0,25, \\ -5 \times 10^{-4}\end{array} & \end{array}$

\section{Control del VIH-1}

Generalmente el método de Runge Kutta de cuarto orden se utiliza para ecuaciones diferenciales no lineales ya que la convergencia estabilidad y consistencia está garantizada, veamos una aplicación a Sistemas Dinámicos no Lineales definidas en $I^{3}$.

$\mathrm{X}(\mathrm{t}) \in \mathbb{R ^ { 3 }}$ y $X^{\prime} \in \mathbb{I}^{3}$ tal que $X^{\prime}(t)=f(X(t))$.

$X^{\prime}=\left(\begin{array}{c}\mathrm{x}_{1}{ }^{\prime} \\ \mathrm{x}_{2}{ }^{\prime} \\ \mathrm{x}_{3}{ }^{\prime}\end{array}\right)$

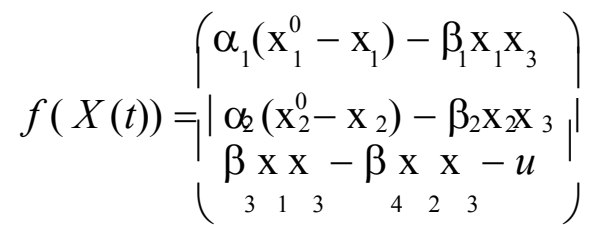

Aplicando el Método de Runge Kutta para problemas de valor inicial asociado a sistemas Dinámicos no lineales [9, 10 y 11], se considera parámetros vectoriales:

$K_{1}, K_{2}, K_{3}$ y $K_{4} \in \mathbb{I}^{3}$, puesto que depende de luego tenemos:

$$
\begin{aligned}
& K_{1}=h\left[f\left(X\left(t_{n}\right)\right)\right] \\
& K_{2}=h\left[f\left(X\left(t_{n+1 / 2}\right)+K_{1} / 2\right)\right] \\
& K_{3}=h\left[f\left(X\left(t_{n+1 / 2}\right)+K_{2} / 2\right)\right] \\
& \left.K_{4}=h\left[f\left(X\left(t_{n+1}\right)+K_{3}\right)\right)\right] \\
& X\left(t_{n+1}\right)=X\left(t_{n}\right)+1 / 6\left[K_{1}+2 K_{2}+2 K_{3}+K_{4}\right]
\end{aligned}
$$

\section{Algoritmo de control}

El algoritmo del esquema numérico con el método de Runge Kutta de cuarto orden que resuelven los Sistemas Dinámicos no Lineales para el modelo propuesto, cuyos resultados se obtienen para $\mathrm{u}=0$ que corresponden al avance de la enfermedad sin medicación y con $\mathrm{u} \neq 0$ cuando el paciente se encuentra en tratamiento antirretroviral, obedece al siguiente proceso:

- Inicio: $\mathrm{t}_{0}, \mathrm{t}_{\mathrm{n}}, \mathrm{w}_{0}$ donde $\mathrm{w}_{0}=\left(\mathrm{x}_{1}{ }^{0}, \mathrm{x}_{2}{ }^{0}, \mathrm{x}_{3}{ }^{0}\right)$.

- $\quad \underline{\text { Paso 1. }}$ - Calculando $h=\frac{t_{n}-t_{0}}{n} \quad \mathrm{y}$ sabiendo que $\mathrm{w}\left(\mathrm{t}_{0}\right)=\mathrm{w}_{0}$.

- Paso 2.- Desde $\mathrm{j}=0,1, . ., \mathrm{n}$ hacer

- Paso 3. $-\mathrm{t}_{\mathrm{j}}=\mathrm{t}_{0}+\mathrm{jh}$

- $\quad$ Paso 4.- $\mathrm{K}_{1}=\mathrm{hF}\left(\mathrm{w}_{\mathrm{j}}\left(\mathrm{t}_{\mathrm{j}}\right)\right)$ 
- Paso 5. $-\mathrm{K}_{2}=\mathrm{hF}\left(\mathrm{w}_{\mathrm{j}}\left(\mathrm{t}_{\mathrm{j}}+\mathrm{h} / 2\right)+\mathrm{K}_{1} / 2\right)$

- Paso 6. $-\mathrm{K}_{3}=\mathrm{hF}\left(\mathrm{w}_{\mathrm{j}}\left(\mathrm{t}_{\mathrm{j}}+\mathrm{h} / 2\right)+\mathrm{K}_{2} / 2\right)$

- $\quad$ Paso 7.- $\mathrm{K}_{4}=\mathrm{hF}\left(\mathrm{w}_{\mathrm{j}}\left(\mathrm{t}_{\mathrm{j}}+\mathrm{h}\right)+\mathrm{K}_{3}\right)$

- Paso8.- $\mathrm{w}_{\mathrm{j}+1}=\mathrm{w}_{\mathrm{j}}\left(\mathrm{t}_{\mathrm{j}}\right)+\mathrm{h} / 6\left(\mathrm{~K}_{1}+2 \mathrm{~K}_{2}+2 \mathrm{~K}_{3}+\mathrm{K}_{4}\right)$, Donde $\mathrm{w}=\left(\mathrm{x}^{\mathrm{j}}{ }_{1}, \mathrm{x}^{\mathrm{j}}{ }_{2}, \mathrm{x}_{3}{ }^{\mathrm{j}}\right)$

- $\quad \underline{\text { Paso }}$. $. j=j+1$

Donde: $\quad \mathrm{K}_{1}=\left(\mathrm{K}_{1}{ }^{1}, \mathrm{~K}_{2}{ }^{1}, \mathrm{~K}_{3} \quad\right)^{1}, \quad \mathrm{~K}=\left(\begin{array}{llll}\mathrm{K}_{1} & , \mathrm{~K}_{2} & { }^{2} \mathrm{~K}_{3} & \\ & \end{array}\right)$, $\mathrm{K}_{3}=\left(\mathrm{K}_{1}{ }^{3}, \mathrm{~K}_{2}{ }^{3}, \mathrm{~K}_{3}{ }^{3}\right), \mathrm{K}_{4}=\left(\mathrm{K}_{1}{ }^{4}, \mathrm{~K}_{2}{ }^{4}, \mathrm{~K}_{3}{ }^{4}\right)$.

\section{RESULTADOS NUMÉRICOS}

Las gráficas de los resultados del Sistema Dinámico no Lineal del VIH-1, se muestran a continuación: cuando $\mathrm{u}=0$, en la Figura 1 , se observa la variación de la cantidad de Linfocitos $\mathrm{T}$ "Helpers" (CD4), representada por $\mathrm{x}_{1}(\mathrm{t})$, por ejemplo para $\mathrm{t}=20$, es casi constante.

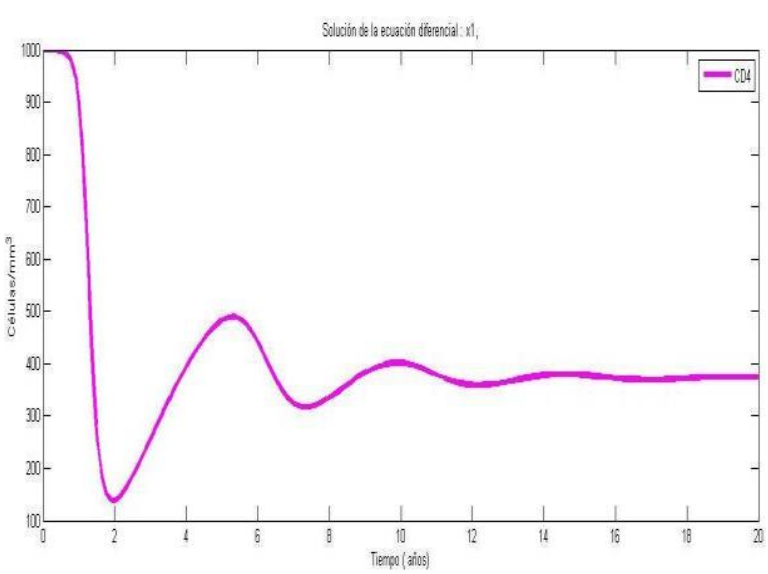

Fig.1 Solución numérica de $x_{1}(t)$ en el intervalo de tiempo $[0,20]$.

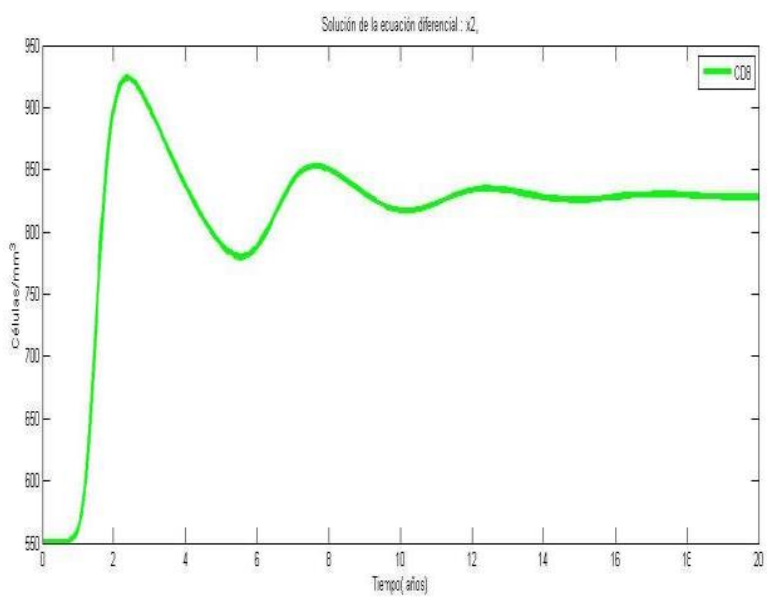

Fig. 2 Solución numérica de $x_{2}(t)$ en el plano $t$ vs $x_{2}$.

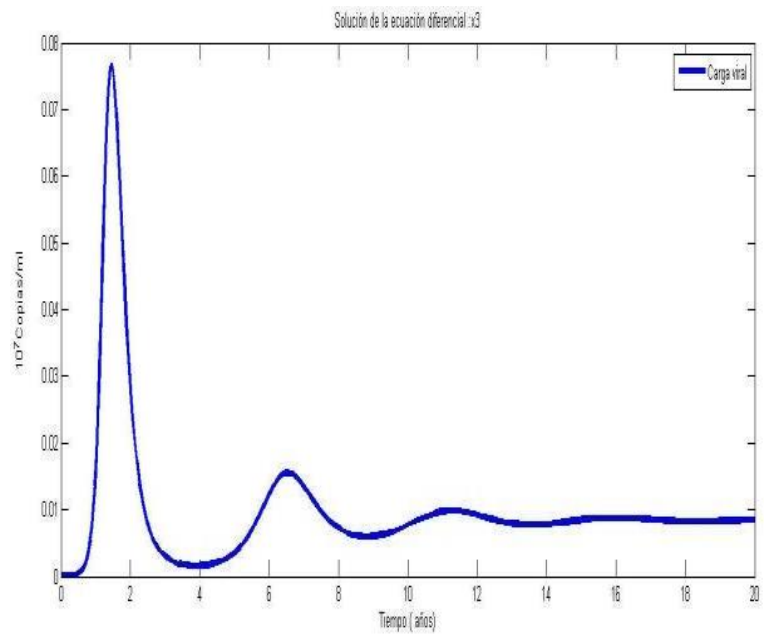

Fig. 3 Solución numérica de $x_{3}(t)$ en el plano $t v s$ $x_{3}$.

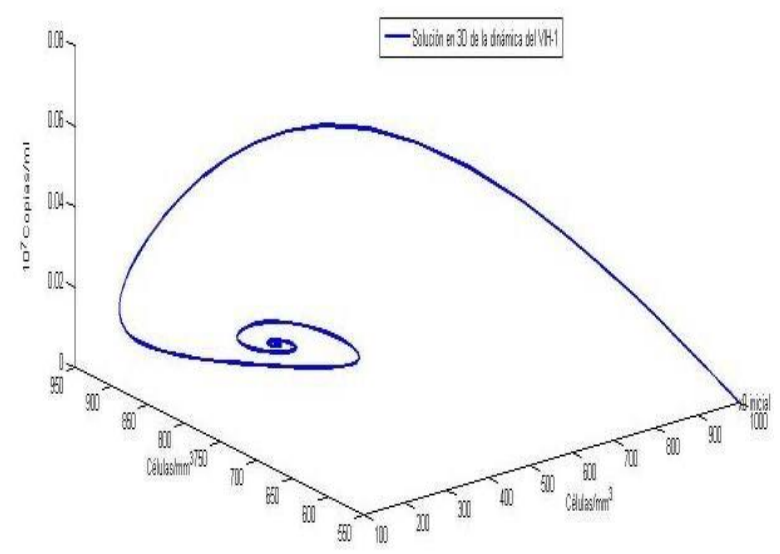

Fig. 4 Solución numérica en $3 D$.

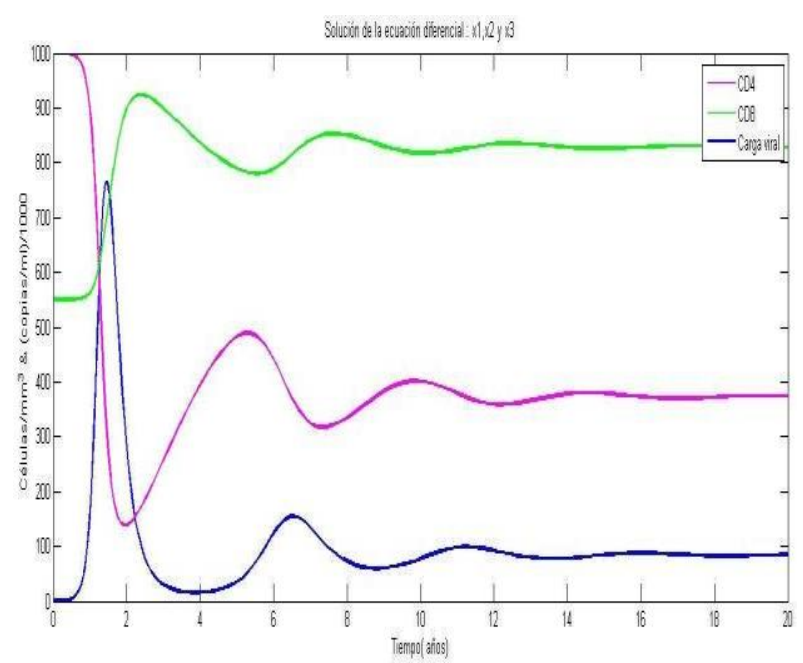

Fig. 5 Primera intersección de soluciones de $x_{1} y$ $x_{2}$ sin tratamiento $(u=0)$. 
En las Figuras 2, 3 y 4 se observa que a partir de un punto de no infección (inestable) que es punto de condición inicial, que también es el primer punto de equilibrio y que se dirige a un punto de infección (estable) que es el segundo punto de equilibrio indicado en el cuadro (1).

En la Figura 5 podemos ver que la intersección de $\mathrm{x}_{1} \mathrm{y} \mathrm{x}_{2}$, esta indica que las concentraciones de CD4 y CD8 son decreciente y creciente respectivamente hasta llegar a una estabilidad que empieza al 8vo año.

Las soluciones del Sistema Dinámico no Lineal del VIH-1 cuando $u \neq 0$, lo podemos observar en la Fig. 6.

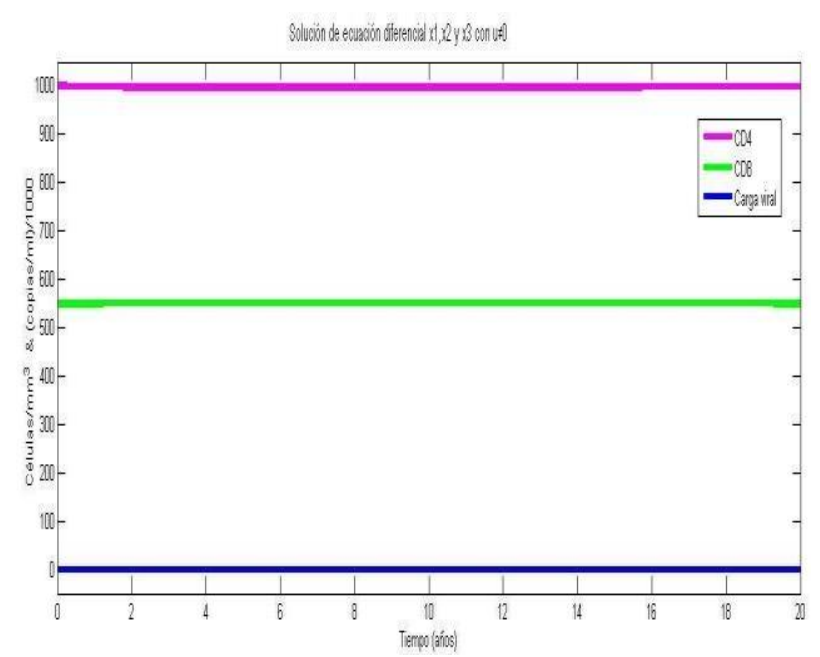

Fig. 6 El CD4, CD8 y Carga Viral (V) que son la solución del Sistema Dinámico del VIH con tratamiento $(u \neq 0)$.

A partir de un punto de no infección estable (primer punto de equilibrio) a un punto de no infección estable (segundo punto de equilibrio y también que las concentraciones de CD4 y CD8 se mantienen estables, es decir, estos valores permanecen iguales a las condiciones iniciales durante el tratamiento antirretroviral.

A continuación se puede observar el comportamiento del CD4 y CD8 para un paciente (con C.I. de Infección) 372.92 células $/ \mathrm{mm}^{3}, 828.7$ células $/ \mathrm{mm}^{3}$ y $0.1 \times 10^{7}$ copias $/ \mathrm{ml}$ en un período de un año de tratamiento. Ver Fig. 7 y 8.

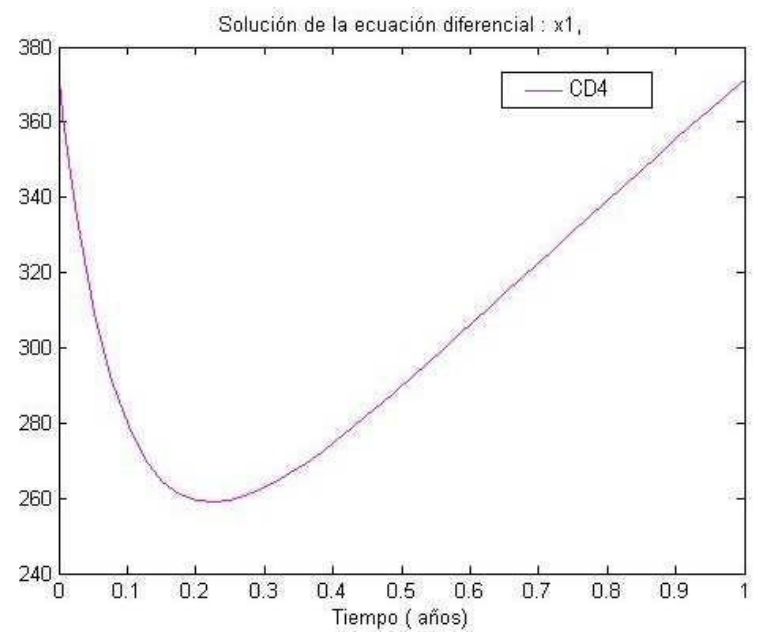

Fig. 7 Solución numérica de $x_{1}$ al año de tratamiento.

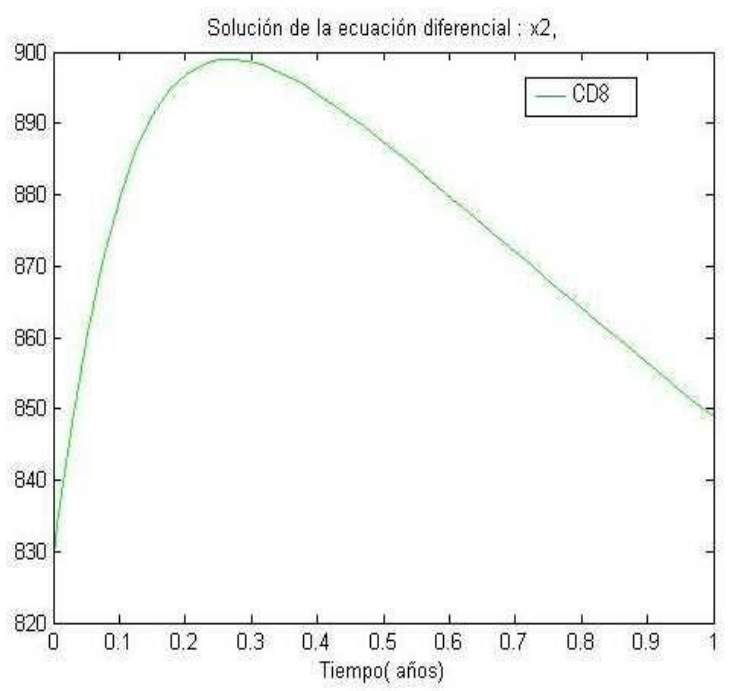

Fig. 8 Solución numérica de $x_{2}$ al año de tratamiento.

Vemos que a partir del segundo mes aproximadamente el CD8 va disminuyendo.

En las Figuras 9, 10, 11 y 12 se muestra el comportamiento del CD4 y CD8 para un paciente con 372.92 células $/ \mathrm{mm}^{3}, 828.7$ células $/ \mathrm{mm}^{3}$ y 0.1 $\mathrm{x} 10^{7}$ copias $/ \mathrm{ml}$ en un período de 16 años. 


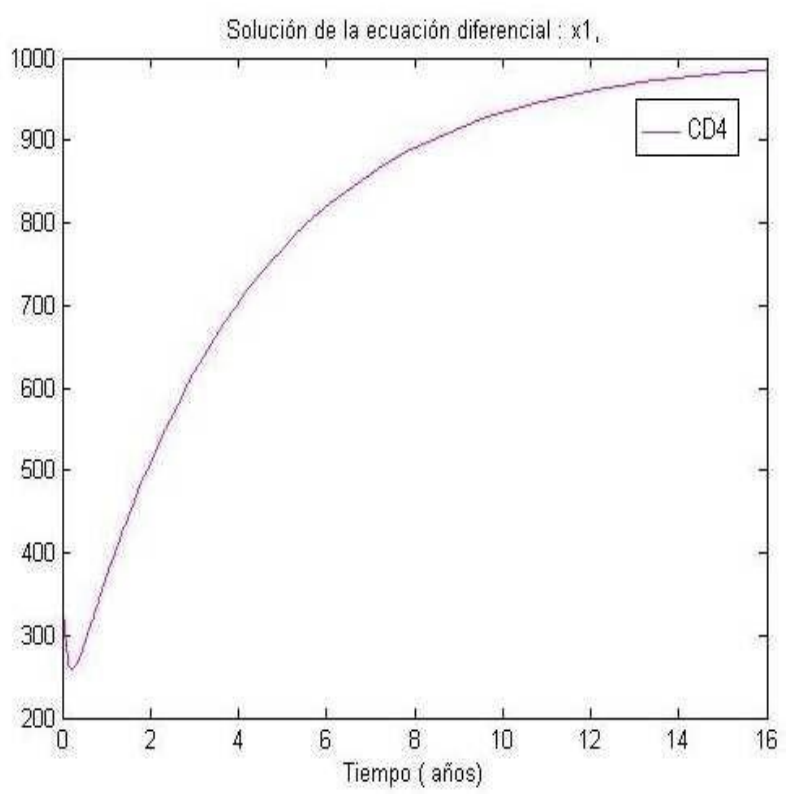

Fig. 9 Solución numérica de $x_{1}$ en 16 años de tratamiento.

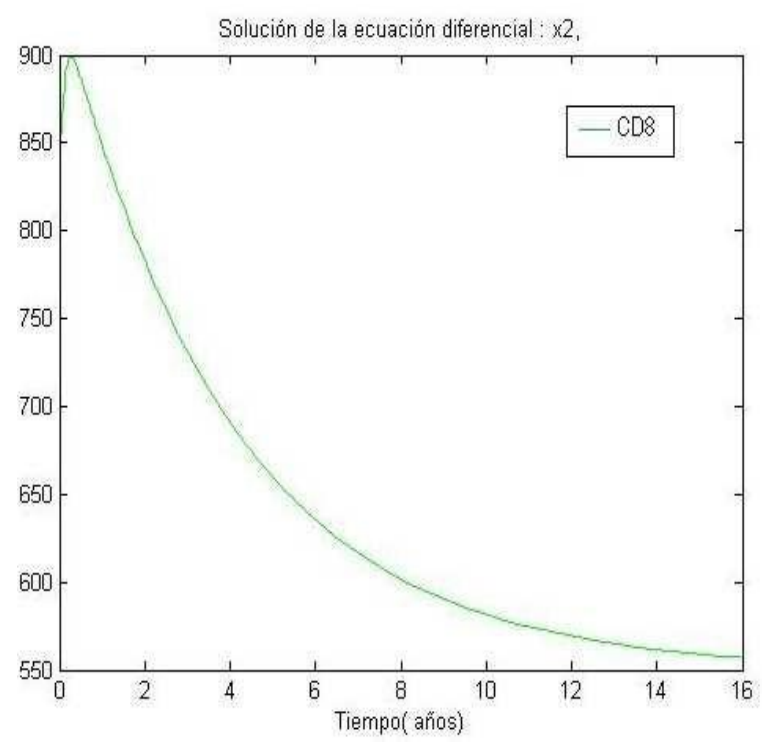

Fig. 10 Solución numérica de $x_{2}$ en 16 años de tratamiento.

Se observa en las gráficas que el CD4 y CD8 va incrementando y disminuyendo respectivamente hasta aproximarse a sus valores normales, y la carga viral es casi indetectable.

\section{CONCLUSIONES}

En el estudio cualitativo se ha considerado la aplicación del Teorema de Grobman - Hartman para garantizar que las soluciones del caso lineal y

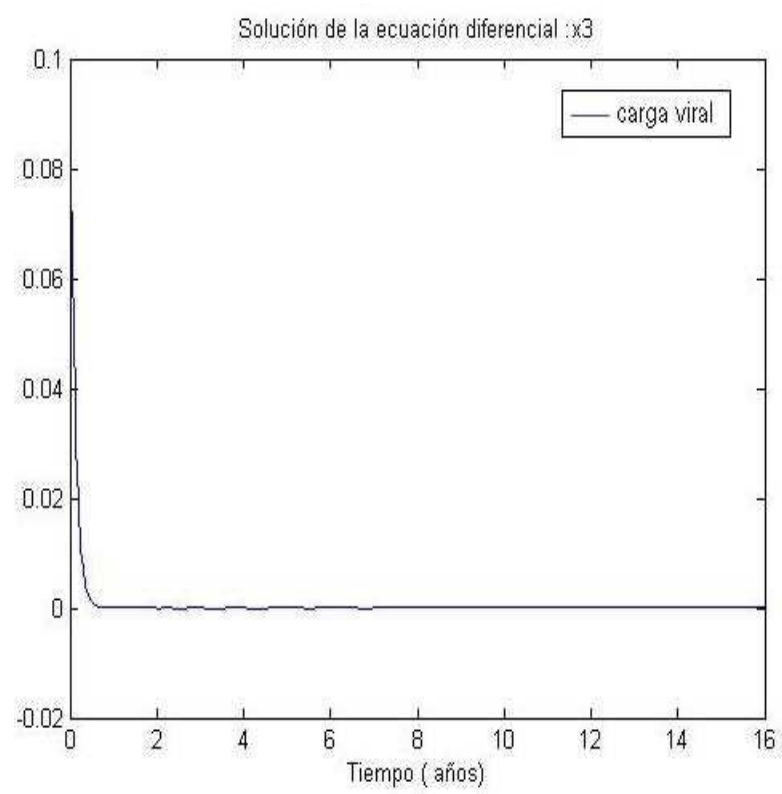

Fig. 11 Solución numérica de $x_{3}$ en 16 años de tratamiento.

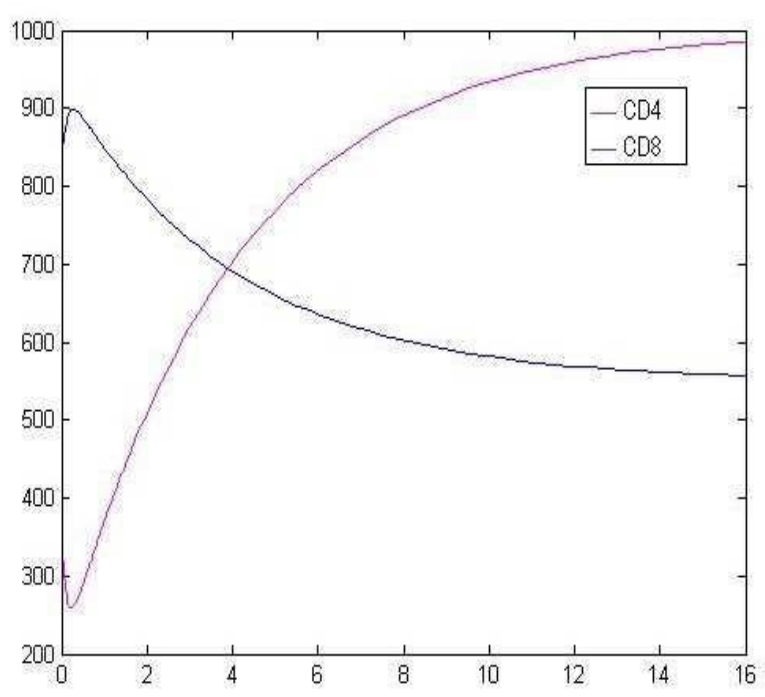

Fig. 12 Solución numérica de $x_{1}(t)$ y $x_{2}(t)$ en 16 años de tratamiento.

no lineal son conjugadas $\mathrm{y}$ topológicamente equivalentes.

Desde un punto de vista cuantitativo se ha resuelto el problema No lineal con el Método Numérico de Runge Kutta de 4to orden para el sistema de tres variables y se ha analizado su convergencia usando los criterios de estabilidad y consistencia.

En la Figura 2, se puede notar que la solución del Sistema Dinámico no Lineal para el caso $\mathrm{u}=0$ la 
cual parte de un estado de no infección (primer punto de equilibrio $t=0$ ) que es inestable, hasta el segundo punto de equilibrio (para $\mathrm{t}=8$ aprox.) que sería estable.

A este punto le corresponde la cantidad de CD4 que es 372 células $/ \mathrm{mm}^{3}$, cantidad de CD8 que es 828 células $/ \mathrm{mm}^{3}$ y la carga viral $8.4 \times 10^{4}$ copias $/ \mathrm{ml}$ que corresponde a un punto crítico de la enfermedad.

La búsqueda de este punto permite verificar que la metodología desarrollada para esta evaluación de la enfermedad es eficaz porque nos permitió encontrar dicho punto que corresponde a $\mathrm{t}=8$ años aprox., donde se sugiere empezar el tratamiento antirretroviral.

En la solución del Sistema Dinámico no Lineal cuando se adiciona $\mathrm{u}=\mathrm{kx}_{3}$ (uso de antirretrovirales), se puede observar que al cabo de un año con una estricta dosis de antirretrovirales, el paciente recupera la cantidad de CD4 y de CD8 de una persona sana entonces la carga viral ya es indetectable en un mayor intervalo de tiempo. Este comportamiento lo podemos ver en las Figura 7 y Figura 8.

Para un paciente con 372.92 células $/ \mathrm{mm}^{3}, 828.7$ células $/ \mathrm{mm}^{3}$ y 0.1 copias $/ \mathrm{ml}$, se observa que a partir del segundo mes aproximadamente de tratamiento, el CD4 empieza su crecimiento y el CD8 empieza su decrecimiento. Estos valores se acercan a 1000 células $/ \mathrm{mm}^{3}, 550$ células $/ \mathrm{mm}^{3}$ y 0 copias $/ \mathrm{ml}$ respectivamente en un período de tiempo de 16 años, este comportamiento se observa en la Fig. 9, 10, y 11.

En tal sentido desde el punto de vista matemático, según los resultados obtenidos se puede ver que se ajusta a la realidad la simulación numérica realizada del proceso infección - tratamiento, donde se puede observar que se requiere de un control estricto del tratamiento en un paciente de VIH-1, hasta ver la eliminación del virus en el la sangre del paciente.

\section{AGRADECIMIENTOS}

Los autores agradecen al Instituto General de Investigación de la Universidad Nacional de
Ingeniería, por haber apoyado con el financiamiento para la ejecución de este proyecto de investigación desarrollado en el Laboratorio de Simulación e Investigación Numérica de la Facultad de Ciencias-UNI.

\section{REFERENCIAS}

1. Freeman, Scott. "Biological Science. Upper Saddle River", NJ: Prentice Hall, (2002).

2. Campos, S. S Ge, Z. Tian., T. H. Lee, "Nonlinear Control of a Dynamic Model of HIV-1", IEEE transactions, Vol. 52, No. 3, pp. 353-361, Marzo (2005).

3. Campello de Sousa, F. M., "Modeling the Dynamics of HIV-1 and CD4 and CD8 Lymphocytes", IEEE Engineering in Medicine and Biology, pp.21-24, January/February (1999).

4. Soto Ramírez, L. E., "et al. Guía de manejo antirretroviral de las personas con VIH". cuarta edición. México (2009).

5. Dirección General de epidemiológica situación del VHI/SIDA en el Perú. "Boletín Epidemológico mensual". Marzo (2012).

6. Chertorivski Woldenberg, S., "et al. Manual de procedimientos Estandarizados para la Vigilancia Epideomológica del VIH-SIDA". Dirección General de Epidemiología. México (2009).

7. Benazic, R., "Tópicos de Ecuaciones Diferenciales Ordinarias". (2007).

8. Mantilla, I., Masgo, J. C. "Teorema de Grobman-Hartman en Espacios de Banach", Seminario de Matemática Pura y Aplicada I, Facultad de Ciencias, Universidad Nacional de Ingeniería (2012).

9. Mantilla, I., Masgo, J. C., "Comportamiento de Especies en Competencia, modelo Matemático. Seminario de Matemática Pura y Aplicada II, Facultad de Ciencias, Universidad Nacional de Ingeniería (2012).

10. Burden, Richard L., Douglas Faires, J., Análisis Numérico (2001).

11. Nakamura, S., "Análisis numérico y visualización grafica con MATLAB”. (1997).

Correspondencia: irlamn@uni.edu.pe

Recepción de originales: febrero 2015

Aceptación de originales: abril 2015 